\title{
Anomalous Hall effect in Fe/Au multilayers
}

\author{
Q. Zhang, ${ }^{1}$ P. Li, ${ }^{1,2}$ Y. Wen, ${ }^{1}$ C. Zhao, ${ }^{3}$ J. W. Zhang, ${ }^{1,4}$ A. Manchon, ${ }^{1}$ W. B. Mi, ${ }^{2}$ Y. Peng,,${ }^{1,4,}{ }^{*}$ and X. X. Zhang ${ }^{1, \dagger}$ \\ ${ }^{1}$ Physical Science and Engineering Division (PSE), King Abdullah University of Science and Technology (KAUST), \\ Thuwal 23955-6900, Saudi Arabia \\ ${ }^{2}$ Tianjin Key Laboratory of Low Dimensional Materials Physics and Preparing Technology, Institute of Advanced Materials Physics, \\ Faculty of Science, Tianjin University, Tianjin 300072, People's Republic of China \\ ${ }^{3}$ Imaging and Characterization Core Lab, King Abdullah University of Science and Technology (KAUST), Thuwal 23955-6900, Saudi Arabia \\ ${ }^{4}$ Key Laboratory of Magnetism and Magnetic Materials of Ministry of Education, Lanzhou University, Lanzhou 730000, \\ Gansu, People's Republic of China
}

(Received 12 January 2016; revised manuscript received 26 May 2016; published 22 July 2016)

\begin{abstract}
To understand the interfacial scattering effect on the anomalous Hall effect (AHE), we prepared multilayers of $\left(\mathrm{Fe}_{(36 / n) \mathrm{nm}} / \mathrm{Au}_{(12 / n) \mathrm{nm}}\right)_{n}$ using an e-beam evaporator. This structure design allowed us to investigate the effect of interfacial scattering on the AHE, while keeping the samples' thickness and composition unchanged. We measured the (magneto)transport properties of the samples in a wide temperature range (10-310 K) with magnetic fields up to $50 \mathrm{kOe}$. We found that the scaling between the anomalous Hall resistivity $\left(\rho_{\mathrm{AHE}}\right)$ and longitudinal resistivity $\left(\rho_{x x}\right)$ can be roughly described by $\rho_{\mathrm{AHE}} \sim \rho_{x x}^{\gamma}$ with $\gamma=2.65 \pm 0.10$ and $1.90 \pm 0.04$ for samples from $n=1$ to $n=4$ and samples from $n=4$ to $n=12$, respectively. Our quantitative analysis results showed that the interfacial scattering suppresses the contribution of the intrinsic mechanism and gives rise to a side-jump contribution.
\end{abstract}

DOI: 10.1103/PhysRevB.94.024428

\section{INTRODUCTION}

The anomalous Hall effect (AHE) in ferromagnetic materials was discovered more than one century ago [1]. Although the AHE has been studied for a long time, and particularly extensively in the past two decades, its origin(s)/mechanism(s) have not been conclusively determined. Theories of both intrinsic and extrinsic mechanisms were proposed to interpret the experimental observations [2-4]. The intrinsic mechanism theory [2] suggests that the AHE originates from spin-orbit coupling in Bloch bands in a perfect ferromagnetic crystal and that there is a quadratic dependence of the anomalous Hall resistivity $\left(\rho_{\mathrm{AHE}}\right)$ on the longitudinal resistivity $\left(\rho_{x x}\right)$, i.e., $\rho_{\mathrm{AHE}} \sim \rho_{x x}^{2}$. Skew scattering [3] and side jump [4] were proposed by Smit and Berger, respectively, as extrinsic mechanisms, ascribing the AHE to electron scattering from imperfections in ferromagnetic materials. The scaling between $\rho_{\mathrm{AHE}}$ and $\rho_{x x}$ was derived as $\rho_{\mathrm{AHE}} \sim \rho_{x x}$ and $\rho_{\mathrm{AHE}} \sim \rho_{x x}^{2}$ for skew scattering and side jump, respectively. Interestingly, the scaling of $\rho_{\mathrm{AHE}} \sim \rho_{x x}^{2}$ is valid for both the intrinsic and extrinsic (side-jump) mechanisms, a controversy that remains unresolved, in addition to the question of whether skew scattering or side jump dominates in the AHE.

Studying how imperfections/scatterings in the material affect the AHE should reduce the ambiguity and improve understanding of the mechanism(s) of the AHE. To this end, interfacial scattering in granular [5,6] and multilayer $[7,8]$ systems and surface scattering [9-11] have been used to tune the longitudinal and anomalous Hall resistivity, experimentally. However, considering the side jump, the commonly used scaling law $\rho_{\mathrm{AHE}} \sim \rho_{x x}^{\gamma}$ is valid only for relatively homogeneous bulklike systems and invalid when the layer

\footnotetext{
*Corresponding author: pengy@lzu.edu.cn

†Corresponding author: xixiang.zhang@kaust.edu.sa
}

thickness is of the order of or smaller than the mean free path (MFP) in multilayers [12]. For inhomogeneous granular and multilayer samples, spin-dependent interfacial scattering leads to large $\gamma(>2)$, e.g., $\gamma=2.6$ in Fe/Cr multilayers [13] and $\gamma=3.7$ in Co-Ag granular thin films [14], which cannot be ascribed to any of the three mechanisms above. Moreover, in the multilayer heterostructures, the shunting effect is a critical issue in Hall resistivity measurement [15-17]. Because the Hall voltage depends on the current flow in each layer, the conventional bulk scaling law cannot be applied for samples with different current distributions.

Another issue is the difficulty of distinguishing the contributions of the intrinsic and side-jump mechanisms experimentally because both mechanisms are predicted to display the same scaling law. Recently, significant progress was achieved by proposing a new scaling relation [9]:

$$
\rho_{\mathrm{AHE}}=\left(\alpha \rho_{x x 0}+\beta \rho_{x x 0}^{2}\right)+b \rho_{x x}^{2},
$$

or, in the form of conductivity,

$$
\sigma_{\mathrm{AHE}}=\left(\alpha \sigma_{x x 0}^{-1}+\beta \sigma_{x x 0}^{-2}\right) \sigma_{x x}^{2}+b,
$$

where $\alpha, \beta$, and $b$ represent the contributions from the skew-scattering, side-jump, and intrinsic mechanisms, respectively. The variables $\rho_{x x 0}, \sigma_{\mathrm{AHE}}$, and $\sigma_{x x 0}$ in Eqs. (1) and (2) are, respectively, the residual longitudinal resistivity, the anomalous Hall conductivity, and the residual Hall conductivity. The intrinsic mechanism, skew-scattering, and side-jump contributions were successfully separated using this scaling law in epitaxial Fe [9,18], Ni [10], and Co [11] thin films. In these works, surface scattering in samples with different thicknesses was used to tune the magnetotransport properties. As reported in $\mathrm{Co} / \mathrm{Pd}$ multilayers, surface scattering plays a different role on the AHE compared to interfacial scattering [8,19]. Therefore, it is necessary to examine the impact of spin-orbit-coupled interfacial scattering on the AHE using this scaling law, Eqs. (1) and (2). 


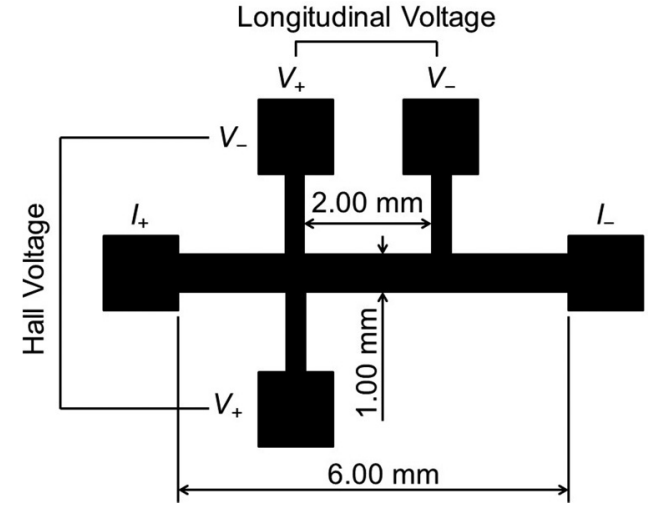

FIG. 1. Schematic diagram of sample (top view) dimensions and measurement configuration for magnetotransport properties.

In the present work, we investigated the AHE in $\left(\mathrm{Fe}_{(36 / n) \mathrm{nm}} / \mathrm{Au}_{(12 / n) \mathrm{nm}}\right)_{n}$ multilayers. We designed this structure based on the following two considerations: First, the total thicknesses of the $\mathrm{Fe}$ and Au layers were kept unchanged in all samples and the current distribution in the $\mathrm{Fe}$ and $\mathrm{Au}$ layers was also the same during the transport measurements, including the Hall resistivity, longitudinal resistivity, and magnetoresistance measurements. The possibility that various current distributions lead to different Hall resistivities can easily be excluded. Second, the total thickness of each sample was the same, such that the scattering contributions from both surfaces would be the same. The only difference in the samples was the number of $\mathrm{Fe} / \mathrm{Au}$ interfaces which is beneficial to the study of the impact of interfacial scattering on the AHE.

\section{EXPERIMENTAL METHODS}

We fabricated multilayers of $\left(\mathrm{Fe}_{(36 / n) \mathrm{nm}} / \mathrm{Au}_{(12 / n) \mathrm{nm}}\right)_{n}(n=$ $1,2,3,4,5,6,8,10,12)$ using an e-beam evaporator (EXPLORER 14, Denton Vacuum). Fe (99.95\% purity) and Au (99.995\% purity) pallets were used as source materials. The samples were deposited, at room temperature, on substrates of oxidized silicon wafers with the base pressure lower than $2.0 \times 10^{-7}$ Torr. The deposition rate $(1.0 \AA / \mathrm{s}$ for both $\mathrm{Fe}$ and $\mathrm{Au}$ layers) and thickness were monitored by a quartz deposition controller (XTC/3S, Inficon). The top layer of each sample was always an Au layer to prevent oxidation. $\mathrm{X}$-ray reflectivity (XRR) and high-angle $\theta-2 \theta$ measurements were carried out using X-ray diffraction (XRD) (D8 DISCOVERY, Bruker) with $\mathrm{Cu} \mathrm{K \alpha}$ radiation. The cross section of samples was characterized by high-resolution transmission electron microscopy (HRTEM) (Titan 80-300, FEI). The longitudinal resistivity, Hall resistivity, and magnetoresistance were measured by a physical property measurement system (PPMS-9, Quantum Design). As shown in Fig. 1, samples were patterned into strips with dimensions $6.0 \mathrm{~mm}$ (length) $\times$ $1.0 \mathrm{~mm}$ (width) $\times 48 \mathrm{~nm}$ (thickness) by mechanical masks for magnetotransport measurement. A five-contact geometry was used for simultaneously measuring the Hall resistivity and longitudinal resistivity on the same piece of sample. A DC constant current of $1000 \mu \mathrm{A}$ was applied during all electrical transport measurements. The sample electrodes were connected to the sample holder of the PPMS with copper wires and the electric contacts were indium disks.

\section{RESULTS AND DISCUSSION}

\section{A. Structural characterizations}

To characterize the multilayered structure of the samples, we ran the XRR measurements on all samples, shown in Fig. 2(a). Characteristic oscillations in the XRR spectra are evident, indicating relatively sharp and flat interfaces. More peaks arising from the superlattice structure are observed in samples with more $\mathrm{Fe} / \mathrm{Au}$ periods (larger $n$ ). To obtain quantitative information about both the periods and individual layer thicknesses of the samples, the XRR spectra were fitted with the LEPTOS software suite (V7.02, Bruker). The fitted thicknesses of each layer were very close to the preset thicknesses for sample preparation, confirming our ability to control the sample fabrication. Figure 2(b) shows the high-angle, $\theta-2 \theta$ XRD patterns for all samples. The samples with few periods or small $n$ exhibited $\mathrm{Fe}(110)$ and $\mathrm{Au}(111)$ peaks, which are the strongest peaks for polycrystalline $\mathrm{Fe}$ and $\mathrm{Au}$, indicating good crystallinity of the Fe and Au layers. As $n$ increased, both peaks became broader and weaker, suggesting that the crystallinity of the samples was decaying. When $n=8-12$, two weak peaks, appearing between the $\mathrm{Fe}(110)$ and $\mathrm{Au}(111)$ peaks, could be due to the FeAu alloy phase formed in the $\mathrm{Fe} / \mathrm{Au}$ interfaces. Because these samples had a clearly layered structure, as suggested by the XRR spectra in Fig. 2(a), the alloy phases could exist only at the interfaces and the interface content in these samples would be quite small.

To further characterize the structure of the multilayer samples and the interfaces between $\mathrm{Fe}$ and Au layers, we analyzed the cross section of selected samples by HRTEM. Figure 3 shows HRTEM images of the cross sections of the multilayers and the corresponding elemental profiles. The HRTEM images clearly exhibit layered structures with relatively sharp interfaces. The rippled structures of the cross sections of multilayers with $n=6$ and 12 could be due to the release of strain, when the $\sim 50$-nm-thick specimens were extracted from the macroscopic samples using a focused ion beam (FIB). The strain was generated at the $\mathrm{Fe} / \mathrm{Au}$ interfaces during growth due to the different lattice constants and thermal expansion coefficients of $\mathrm{Fe}$ and $\mathrm{Au}[20,21]$. This strain release is not evident in samples with small $n$, as indicated in the $n=2$ sample. The rippling of the multilayers caused by strain release does not occur in the macroscopic samples; if it did, we would not observe the low-angle oscillations in the XRR spectra as shown in Fig. 2(a). The measured thickness of each layer and the total thickness of the samples in the HRTEM images are consistent with the preset values, suggesting that the layered structure and thickness of each layer are well controlled during deposition. Elemental profiles were obtained by line scanning of energy-dispersive $\mathrm{x}$-ray spectroscopy (EDX) using a scanning transmission electron microscopy (STEM) as shown in Figs. 3(d), 3(e), and 3(f). As expected, these elemental profiles featuring the desired periodicity demonstrate the high quality of the multilayer samples. 

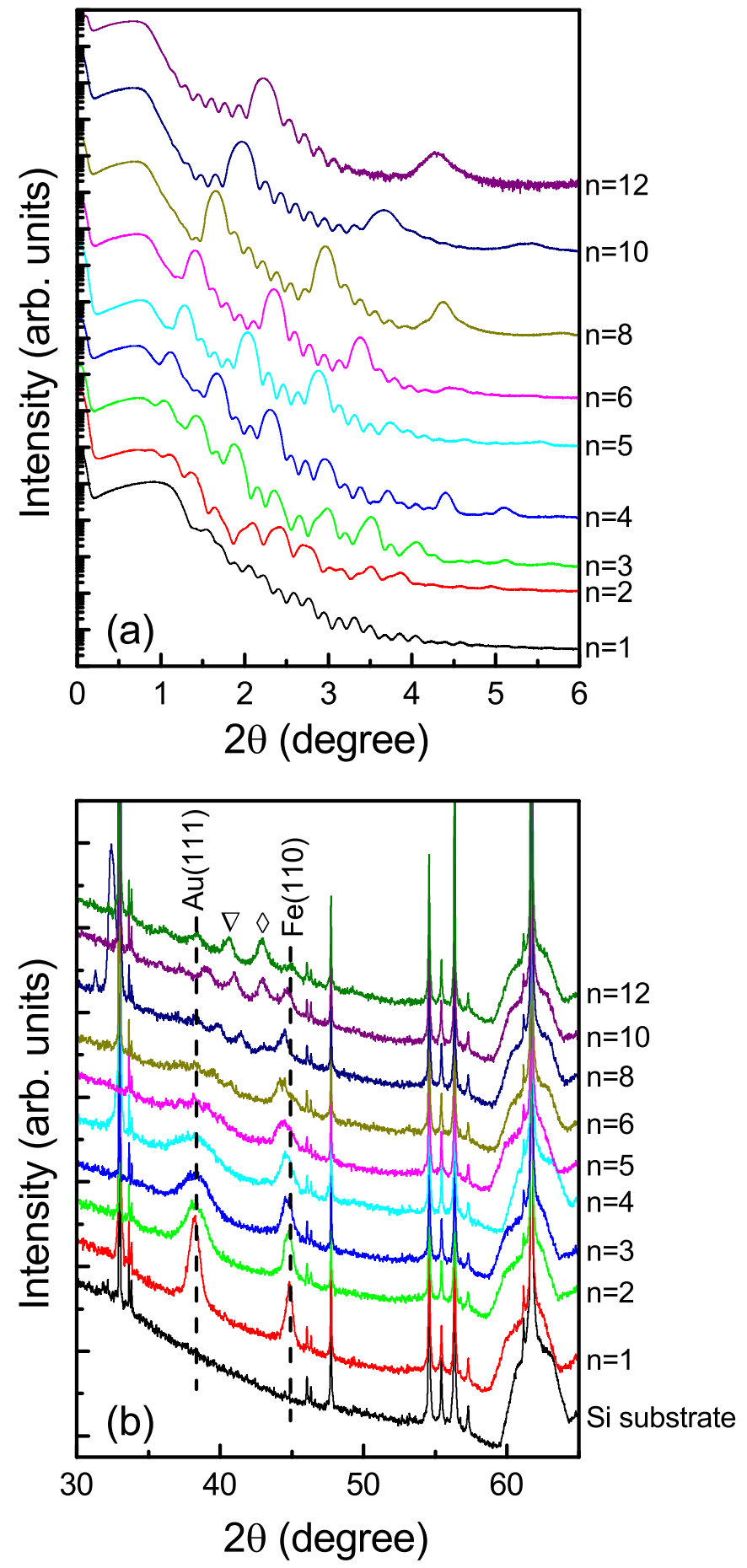

FIG. 2. (a) XRR spectra and (b) high-angle XRD patterns of multilayered samples from $n=1$ to $n=12$. The peaks of $\mathrm{FeAu}$ alloys are labeled as $(\nabla) \operatorname{AuFe}(111)$ and $(\diamond) \operatorname{AuFe}_{9}(111)$ in (b).

\section{B. Longitudinal resistivity}

To understand electrical transport in the multilayered materials, we measured the temperature-dependent longitudinal resistivity at zero magnetic field from 10 to $310 \mathrm{~K}$. Figure 4(a) shows the temperature-dependent $\rho_{x x}$ curves for all samples. The temperature coefficient of resistivity (TCR, $d \rho / d T$ ) was positive for all samples across the full temperature range, indicating the metallic nature of the samples. It is clear that,
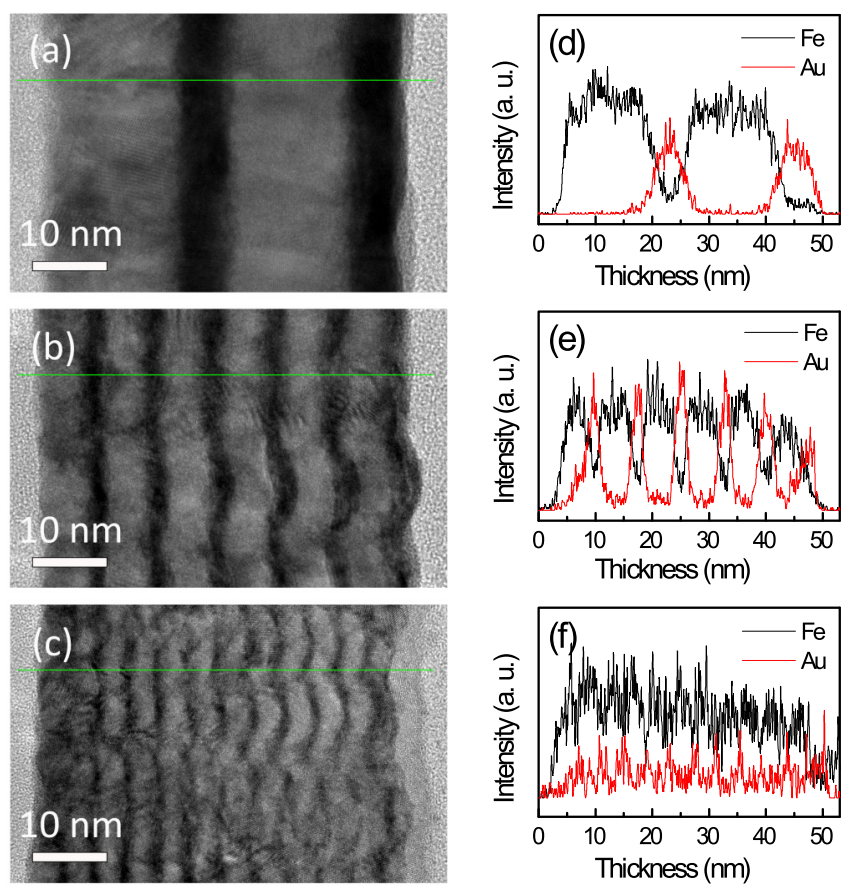

FIG. 3. HRTEM images of cross sections of samples (a) $n=2$, (b) $n=6$, (c) $n=12$ and EDX depth profiles of Fe and Au elements in corresponding samples (d) $n=2$, (e) $n=6$, (f) $n=12$.

as $n$ increases from 1 to 12 , the $\rho_{x x}$ curves shift monotonically to high values, although the total thicknesses of the $\mathrm{Au}$ and $\mathrm{Fe}$ layers remain the same in all samples. The resistivity flattens off below $50 \mathrm{~K}$ in all samples, indicating that the thermal contribution to the total resistivity is less important than the residual resistivity caused by electron scattering by structural defects in both individual layers and the interfaces, particularly at temperatures below $10 \mathrm{~K}$. To better understand the effect of electron scattering by the interfaces on the measured resistivity, we replotted the data of Fig. 4(a) in different forms, as shown in Figs. 4(b)-4(d). One interesting feature in Fig. 4(b) is that the curves of $\rho_{x x}(n) / \rho_{x x}(n=1)$ as a function of temperature might be classified into two groups. The curves with $n \leqslant 4$ are flat across the full temperature range, while the curves for $n \geqslant 5$ are dependent on temperature, the dependence becoming stronger as $n$ increases. This temperature dependence might suggest that the dominant mechanisms of the electron scatterings are different for the two groups of samples. In the $n \leqslant 4$ samples, the contribution from scattering by two-dimensional (2D) interfaces is much weaker than that in the $n \geqslant 5$ samples, although zero-dimensional (0D, relative to the $2 \mathrm{D}$ interfaces) nanometer-sized defects in the individual layers of $\mathrm{Fe}$ and $\mathrm{Au}$ exist in all samples. Because the thickness of the individual layers of $\mathrm{Fe}$ and $\mathrm{Au}$ decreases as $n$ increases, the crystallinity of the samples decays and, consequently the density of the 0D defects, such as grain boundaries, increases. The decaying quality of the crystallinity of the samples with the decreasing thickness of individual layers is reflected in the high-angle XRD spectra shown in Fig. 2(b). The combined electron scattering from the 2D interfaces and OD defects leads to an increase in the contribution of residual resistivity to the total resistivity as reflected in Fig. 4(c), in which 

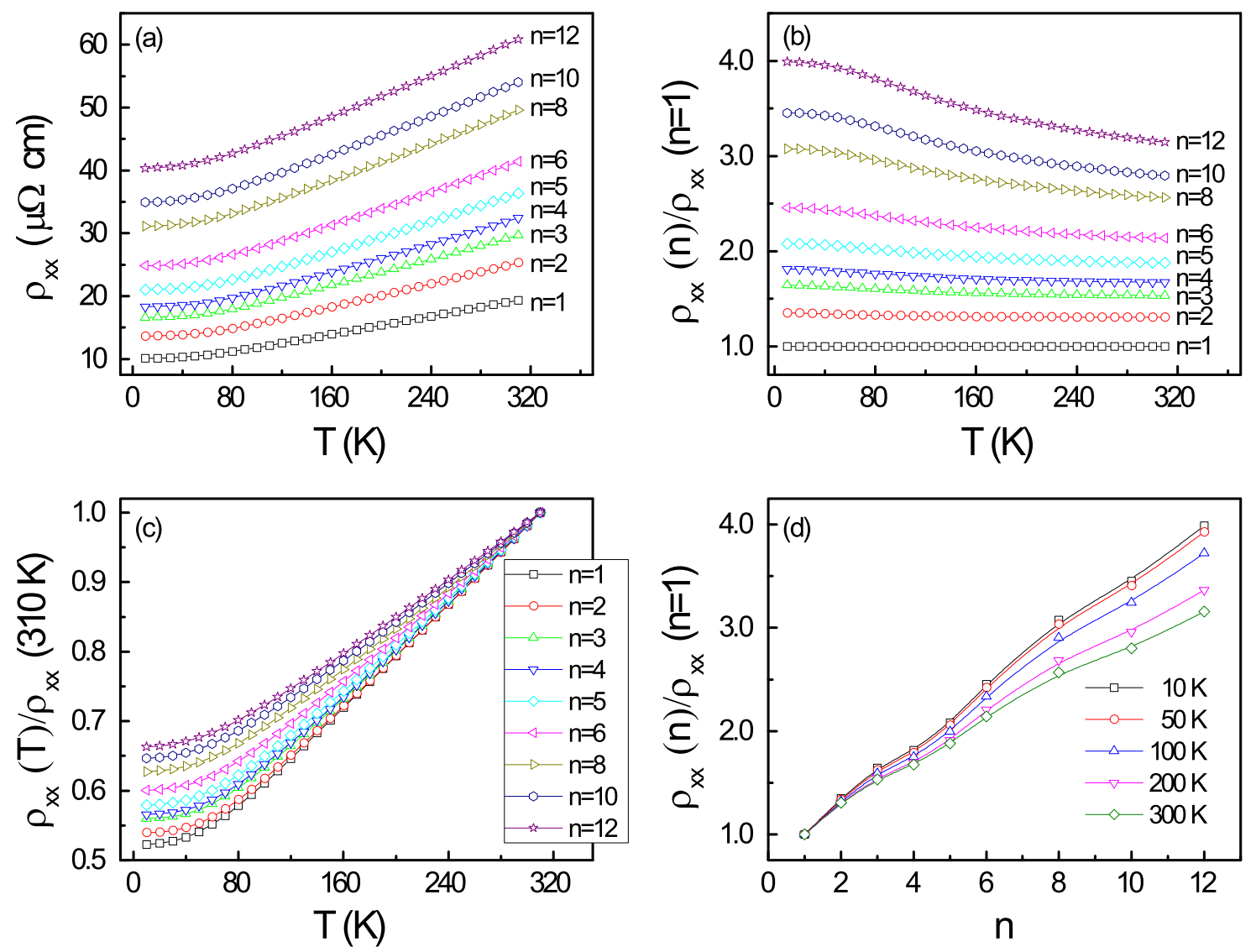

FIG. 4. (a) Temperature-dependent longitudinal resistivity $\left(\rho_{x x}\right)$ curves for all samples; (b) $\rho_{x x}(n) / \rho_{x x}(n=1)$ as a function of temperature for all samples; (c) normalized $\rho_{x x}(T) / \rho_{x x}(310 \mathrm{~K}) \sim T$ curves for all samples; (d) $\rho_{x x}(n) / \rho_{x x}(n=1)$ as a function of number of periods $n$ for selected temperatures.

the $\rho_{x x}(10 \mathrm{~K}) / \rho_{x x}(310 \mathrm{~K})$ ratio increases with $n$. These plots also give information about the relative contributions of both phonons and structural imperfections to the electron scattering. Generally, as the disorder increases, the resistivity decreases more slowly as the temperature decreases [22]. It is therefore evident that the samples with larger $n$ have greater scattering due to the interfaces and poor crystallinity. Due to the size effect, the interfacial scattering plays a more important role in resistivity than does grain boundary scattering for samples with $n \geqslant 5$.

Similar conclusions can be drawn from the plots in Fig. 4(d). It is clear that the $\rho_{x x}(n) / \rho_{x x}(n=1)$ ratios for low-temperature data $(T=10$ and $50 \mathrm{~K})$ in which the phonon scattering can be neglected depend nearly linearly on $n$, which reveals the impact of interfacial scattering. The $\rho_{x x}(n) / \rho_{x x}(n=1)$ ratios for high-temperature data in which the contribution from phonons becomes significant are markedly lower.

\section{Anomalous Hall effect scaling analysis}

Our observations of the relationship between resistivity behaviors and temperature suggest that the $\mathrm{Fe} / \mathrm{Au}$ interface plays a crucial role in electrical transport. We now turn our attention to the AHE to examine how the scattering processes affect the anomalous Hall resistivity. The Hall resistivity $\left(\rho_{x y}\right)$ for all samples was measured with a magnetic field applied perpendicularly to the plane of the films in a range of -5 $\mathrm{T} \leqslant H \leqslant 5 \mathrm{~T}$ and at temperatures ranging from 10 to 300 K. Figure 5(a) shows the field-dependent Hall resistivity of all samples at $10 \mathrm{~K}$. As expected, $\rho_{x y}$ increases sharply with low applied magnetic field and follows a linear dependence up to the magnetic saturation field at which the strongly magnetic-field-dependent Hall resistivity suddenly changes to a much weaker field dependence. This behavior is commonly observed in magnetic thin films that have an easy plane, e.g., the magnetization is lying in the film's plane at zero applied magnetic field. When the magnetic field is applied perpendicularly to the film's plane, magnetic saturation occurs at the demagnetizing field and no hysteresis loops are observed. In Fe thin films, the magnetic saturation field occurs at about $20 \mathrm{kOe}$ and remains almost constant from 10 to $300 \mathrm{~K}$, due to the very high Curie temperature of pure Fe. Therefore, the behaviors of the field-dependent Hall effect and fielddependent magnetization should be very similar, because the anomalous Hall resistivity is proportional to the magnetization component in the field direction as described by

$$
\rho_{x y}=\rho_{\mathrm{OHE}}+\rho_{\mathrm{AHE}}=R_{0} H+R_{s} 4 \pi M,
$$

where $\rho_{\mathrm{OHE}}$ is the normal Hall resistivity arising from the classical Lorentz force and $\rho_{\mathrm{AHE}}$ is the anomalous Hall resistivity originating from the quantum mechanisms. At magnetic fields higher than the saturation field, the weakly field-dependent Hall resistivity should be ascribed to the 

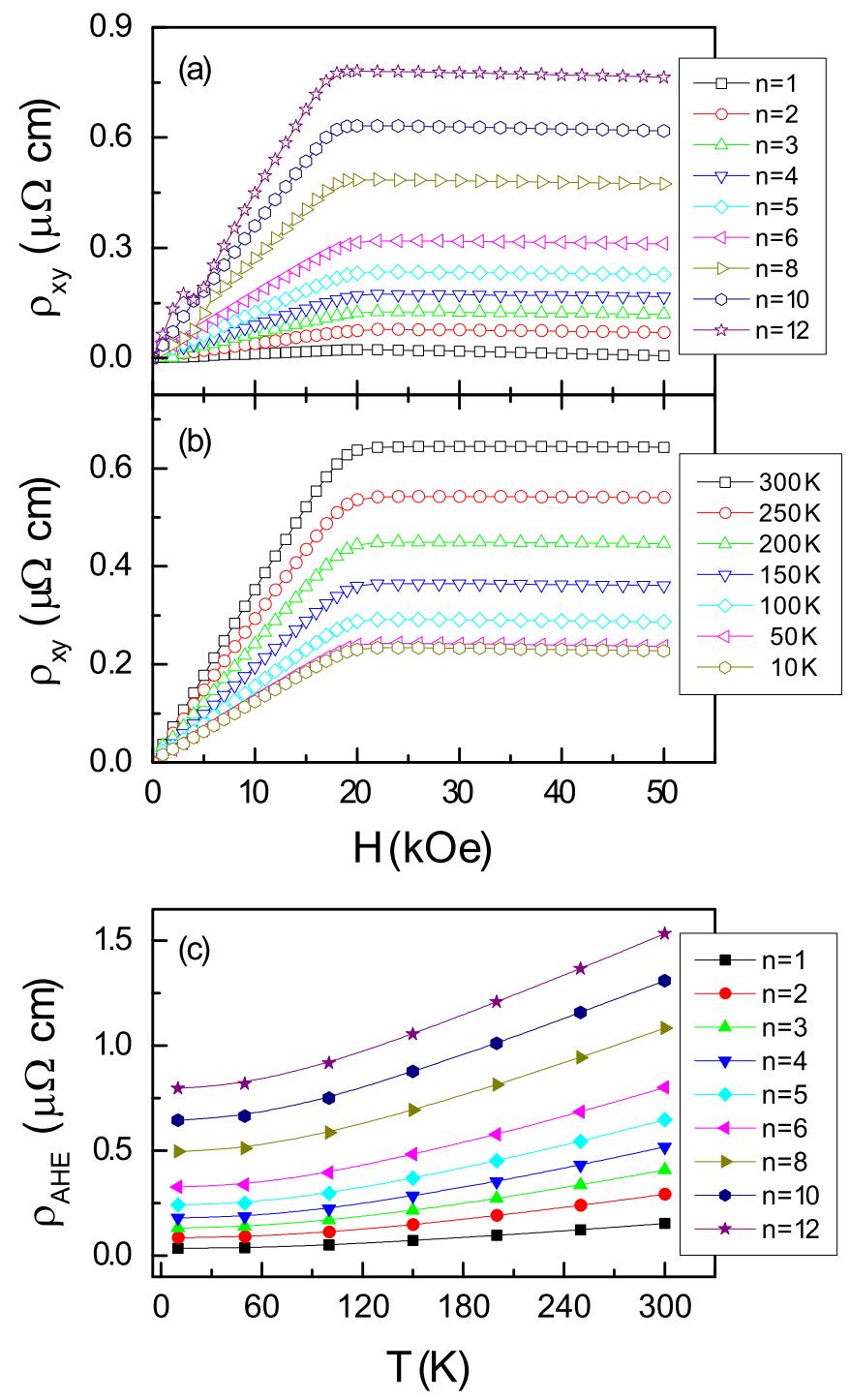

FIG. 5. (a) Field-dependent Hall resistivity $\left(\rho_{x y}\right)$ of all samples at $10 \mathrm{~K}$. (b) Field-dependent Hall resistivity $\left(\rho_{x y}\right)$ of the $n=5$ sample at temperatures ranging from 10 to $300 \mathrm{~K}$. (c) Temperature-dependent anomalous Hall resistivity $\left(\rho_{\mathrm{AHE}}\right)$ for all samples.

contributions from the normal Hall effect and from the slight change in the AHE caused by the improved alignment of the spins. The total magnetization continues to increase in this regime due to the field-forced rotation of the spins against the thermal agitation (except at $0 \mathrm{~K}$ ) and nonferromagnetic couplings. In $\mathrm{Fe}, \mathrm{Co}$, and other ferromagnetic materials that have high Curie temperatures, the slope of the measured Hall resistivity in this region mainly originates from the ordinary Hall effect. In this case, $\rho_{\mathrm{AHE}}$ could be obtained by extrapolating the linear part to zero field.

The sign of $\rho_{\mathrm{AHE}}$ for all samples is positive across the full temperature range of the measurements, which is consistent with the sign of $\rho_{\mathrm{AHE}}$ observed in pure Fe films [9] and $\mathrm{Fe} / \mathrm{Si}$ multilayers [23]. The sign of $\rho_{\mathrm{OHE}}$ is negative, suggesting that electrons dominate the transport properties in all samples. Figure 5(b) shows the field-dependent $\rho_{x y}$ of the sample with $n=5$ measured at different temperatures as an example to illustrate the effect of temperature on the AHE.

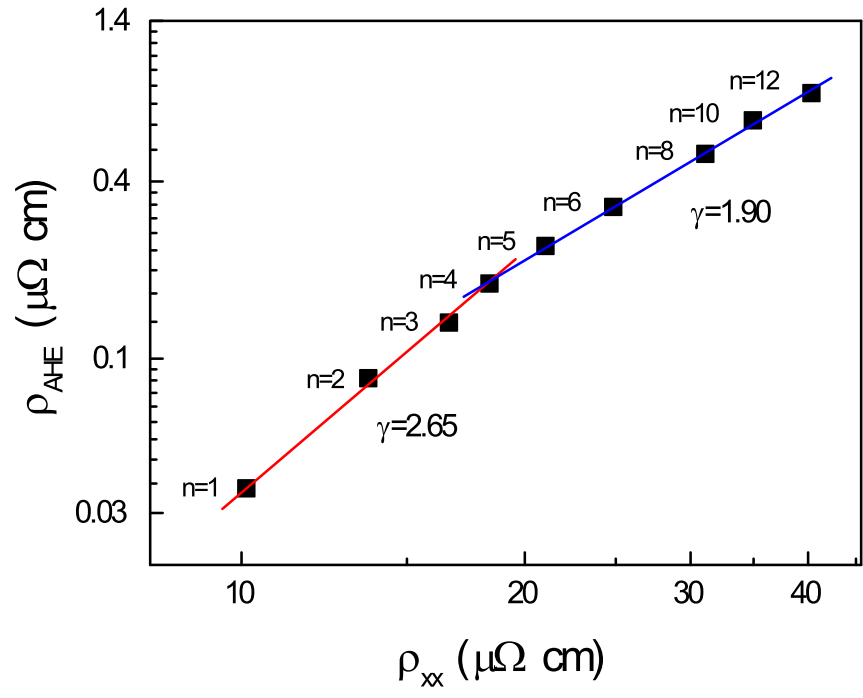

FIG. 6. A logarithmic plot of anomalous Hall resistivity $\left(\rho_{\mathrm{AHE}}\right)$ versus longitudinal resistivity $\left(\rho_{x x}\right)$ measured at $10 \mathrm{~K}$ for all samples. The red and blue lines are linear fits to the data.

As the longitudinal resistivity increases with temperature, $\rho_{x y}$ increases. Figure 5(c) presents the temperature-dependent $\rho_{\mathrm{AHE}}$, which shows the same tendency as $\rho_{x x}(T)$. It clearly shows that $\rho_{\mathrm{AHE}}$ depends very weakly on temperature below $50 \mathrm{~K}$, indicating a quite weak contribution of phonons to the AHE. Remarkably, at $10 \mathrm{~K}, \rho_{\mathrm{AHE}}$ increased by 40 times when $n$ increased from 1 to 12 , although the longitudinal resistivity increased by only four times.

To understand the origin/mechanisms of the AHE experimentally, the relation $\rho_{\mathrm{AHE}} \sim \rho_{x x}^{\gamma}$ is usually employed and the exponent $\gamma$ is then extracted by fitting the experimental data to the power law. As discussed above, $\gamma=1$ indicates that skew scattering is the dominant mechanism, whereas $\gamma=2$ may suggest that an intrinsic mechanism, an extrinsic side-jump mechanism, or both dominate the AHE. The curve of $\rho_{\mathrm{AHE}} \sim \rho_{x x}$ was plotted logarithmically in Fig. 6 for all samples measured at $10 \mathrm{~K}$ at which electron-photon scattering could be neglected. Apparently, not all data can be fitted to a single straight line. A closer analysis reveals that the data in Fig. 6 can be fitted to two linear functions as indicated by the blue and red lines. Fitting the data obtained from the $n=1$ to $n=4$ samples gave $\gamma=2.65 \pm 0.10$, whereas $n=4$ to $n=12$ samples gave $\gamma=1.90 \pm 0.04$. Normally, $\gamma=1.90(\sim 2)$ could be regarded as an intrinsic mechanism, a side-jump mechanism, or both, but it is difficult to distinguish between the two mechanisms conclusively based only on this scaling. When $\gamma$ is larger than 2 , attributing this relation to any of the three mechanisms is difficult. A large exponent has previously been observed in other metallic samples, such as $\mathrm{Fe} / \mathrm{Cr}$ multilayers [13] and $\mathrm{Co}-\mathrm{Ag}$ granular thin films [14,24].

It could be possible that the different exponential components are due to the shunting effect of Au layers in the samples. After reexamining the structures of these samples, this possibility has been excluded in these multilayer structures based on the model shown in Fig. 6 of Ref. [15]. Because an anomalous Hall voltage can be generated only from the Fe layer, the Hall voltage of the multilayers is therefore 

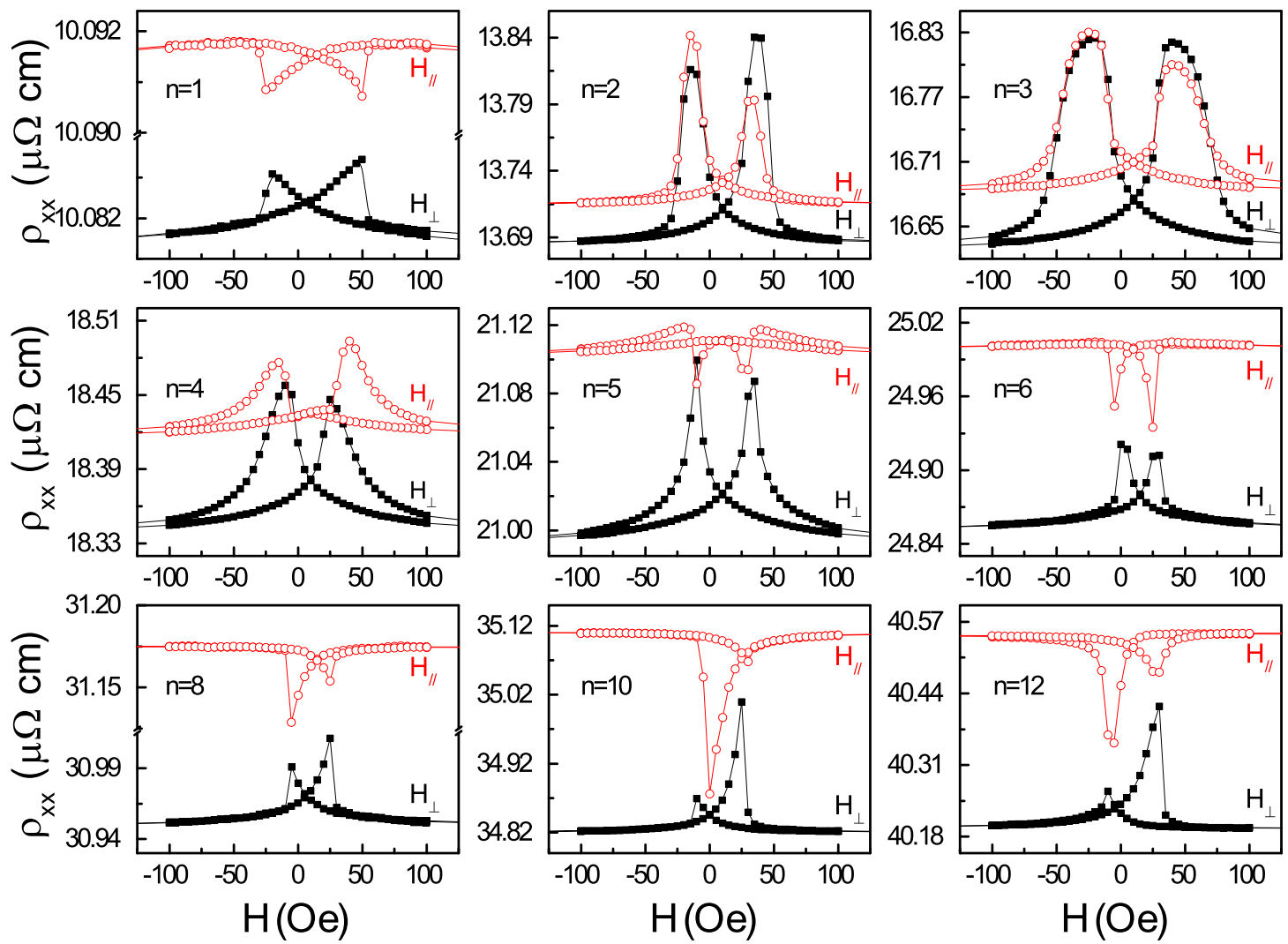

FIG. 7. MR curves of all samples measured at $10 \mathrm{~K} . H_{/ /}\left(H_{\perp}\right)$ indicates that the magnetic field was parallel (perpendicular) to the measurement current. Magnetic field was applied in the sample plane during the measurement.

proportional to that of single Fe layers according to Kirchhoff's law. This proportion remains the same in all samples because the ratio of the longitudinal resistivity $\left(\rho_{x x \mathrm{Fe}} / \rho_{x x \mathrm{Au}}\right)$ and the thickness $\left(t_{\mathrm{Fe}} / t_{\mathrm{Au}}\right)$ between $\mathrm{Fe}$ and Au remains constant in all samples. The scaling law $\rho_{\mathrm{AHE}} \sim \rho_{x x}^{\gamma}$ is therefore not affected by the Au layers.

Whereas the composition (thickness ratio) of $\mathrm{Fe}$ and $\mathrm{Au}$ is exactly the same here, the $\rho_{x x}$ and $\rho_{\mathrm{AHE}}$ are completely different in all samples. The scaling law $\rho_{\mathrm{AHE}} \sim \rho_{x x}^{\gamma}$ gives different $\gamma$ values for samples from $n=1$ to $n=4$ and for samples from $n=4$ to $n=12$. This means that the AHE is directly related to the density and strength of the scattering from the interfaces and grain boundaries. In inhomogeneous systems, like granular thin films, both the composition and the particle size of the nonmagnetic material doped in the samples affect the AHE, which explains why various scaling relations have been reported for very similar granular thin films, e.g., $\mathrm{Fe}_{-} \mathrm{SiO}_{2}$ films, by different groups $[5,25,26]$. In the present work, the influence of composition variation was avoided in the scaling relation and only the amount of interfacial scattering differs between the different samples.

In our previous work [27], giant magnetoresistance (GMR) and anisotropic magnetoresistance (AMR) effects were used to explore the surface/interface scattering in $\mathrm{Co} / \mathrm{Pd}_{1-x} \mathrm{Ag}_{x}$ multilayers. To understand the effect of scattering on transport properties, magnetoresistance (MR) curves were measured with the magnetic field in the sample plane parallel and perpendicular to the current. The GMR effect is based on the two-current model [28] that comes from the spin-dependent scattering both within the ferromagnetic layers and at the interfaces between ferromagnetic and nonferromagnetic layers $[29,30]$. The GMR effect depends on the relative direction of the spins and is not related to the angle between the magnetization and the current. The AMR effect is caused by anisotropic scattering of carriers due to spin-orbit coupling. Generally, it depends on the relative orientations of the magnetization and the current in polycrystalline ferromagnetic materials.

MR curves with in-plane magnetic field perpendicular and parallel to the measurement current are shown in Fig. 7. We found that GMR dominated (the magnitude of the GMR was quite small) in the $n=2,3$ samples. In sample $n=4$, the GMR is comparable to the AMR. AMR dominated in the $n=1$ and $n \geqslant 5$ samples. As reported in Fe/Cr multilayers [13] and Co-Ag granular films [14,24], which are typical GMR systems, the spin-dependent scattering that is responsible for the GMR phenomenon plays an important role in the large exponent $\gamma$ $(>2)$ in the AHE scaling law. This could explain the linear scaling relation with large exponent $(\gamma=2.65 \pm 0.10)$ in the $n=1$ to $n=4$ samples in Fig. 6. The $n=1$ sample is an exception: it did not exhibit the GMR effect because this sample contained only one layer of Fe. In contrast, this sample displayed a prominent AMR effect. In the $n=5$ to $n=12$ samples, AMR dominated the electrical transport, which was related to the spin-orbit coupling that was also the origin of the AHE (either intrinsic or extrinsic AHE). Normally, the GMR should be present in ferromagnet/nonferromagnet 
multilayers, but these samples show only the AMR effect. This could be explained by the interfacial scattering due to large spin-orbit coupling of $\mathrm{Au}$ layers. Let us examine how the interfacial scattering affects the mechanisms of the AHE in the multilayers.

\section{Parsing of intrinsic, skew-scattering, and side-jump contributions}

To distinguish the contributions of the intrinsic mechanism and the side-jump mechanism in the measured AHE, we analyzed our data using the recently proposed scaling [9] as shown in Eqs. (1) and (2). To avoid spin-dependent scattering dominating the transport properties as in GMR materials, we chose the samples with $n=4$ to 12 for this analysis. Since the thermal effect on transport properties can be neglected at low temperature $(10 \mathrm{~K})$, Eq. (2) can then be simplified to $\sigma_{\mathrm{AHE} 0}=\alpha \sigma_{x x 0}+(\beta+b)$ by taking the following approximations: $\sigma_{\mathrm{AHE}} \approx \sigma_{\mathrm{AHE} 0}$ and $\sigma_{x x} \approx \sigma_{x x 0}$. We then plotted $\sigma_{\mathrm{AHE} 0}$ as a function of $\sigma_{x x 0}$ for data obtained at $10 \mathrm{~K}$ in Fig. 8(a). By fitting the data linearly, we extracted the slope $\alpha=(7.69 \pm 4.84) \times 10^{-4}$ and the intercept $\beta+b=$ $(4.02 \pm 0.15) \times 10^{2} \Omega^{-1} \mathrm{~cm}^{-1}$. The relatively small value of $\alpha$ may suggest that the skew scattering, if it exists, contributes very little to the measured AHE. Following the procedure used in Ref. [9], we then plotted the $\sigma_{\mathrm{AHE}}$ curves as a function of $\sigma_{x x}^{2}$ for data obtained across the full temperature range (10 to $300 \mathrm{~K}$ ) for $n \geqslant 4$ samples as shown in Fig. 8(b). By fitting the data to a linear dependence, we obtained the value of $b$ (the intercept) for each sample. Clearly, these curves do not have the same intercept, differing from the results reported in Ref. [9]. The inset of Fig. 8(b) gives the values of $b$ for different samples. Interestingly, the value of $b$ decreases linearly with increasing $n$. Using the value of $\beta+b$ from Fig. 8(a), the value of $\beta$ was easily obtained for each sample. Obviously, $\beta$ is almost zero for $n=4$, suggesting that the intrinsic mechanism dominates the AHE in this sample. Since $\beta+b$ is constant, as $n$ increases from 4 to 12 , the value of $\beta$ increases and, consequently, the value of $b$ decreases [as shown in the inset of Fig. 8(b)], which is indicative of the relative contributions from the intrinsic mechanism and the side-jump mechanism to the AHE, depending on $n$. To understand how both the intrinsic mechanism and side-jump mechanism vary with the scattering properties (density and strength), we plotted both $b$ and $\beta$ in relation to the residual longitudinal resistivity in Fig. 8(c). It is interesting that both $b$ and $\beta$ show a perfectly linear dependence on $\rho_{x x 0}$, although they have opposite trends. This is in sharp contrast to the results reported in Ref. [9]. where both $b$ and $\beta$ remain almost unchanged in single-crystalline Fe films with different thicknesses. In that work, the Fe films have high quality and the mean free path of electrons could be quite large. The surface scattering plays a very important role in electrical transport so the longitudinal resistivity and Hall resistivity were largely tuned by changing the thickness of the $\mathrm{Fe}$ films. In our work, interfacial scattering at $\mathrm{Fe} / \mathrm{Au}$ interfaces plays a more important role than surface scattering does as indicated by the resistivity data in Fig. 4. The original theories suggested that the intrinsic mechanism happens in the perfect crystal [2] and side-jump contribution comes from impurity scattering [4]. We
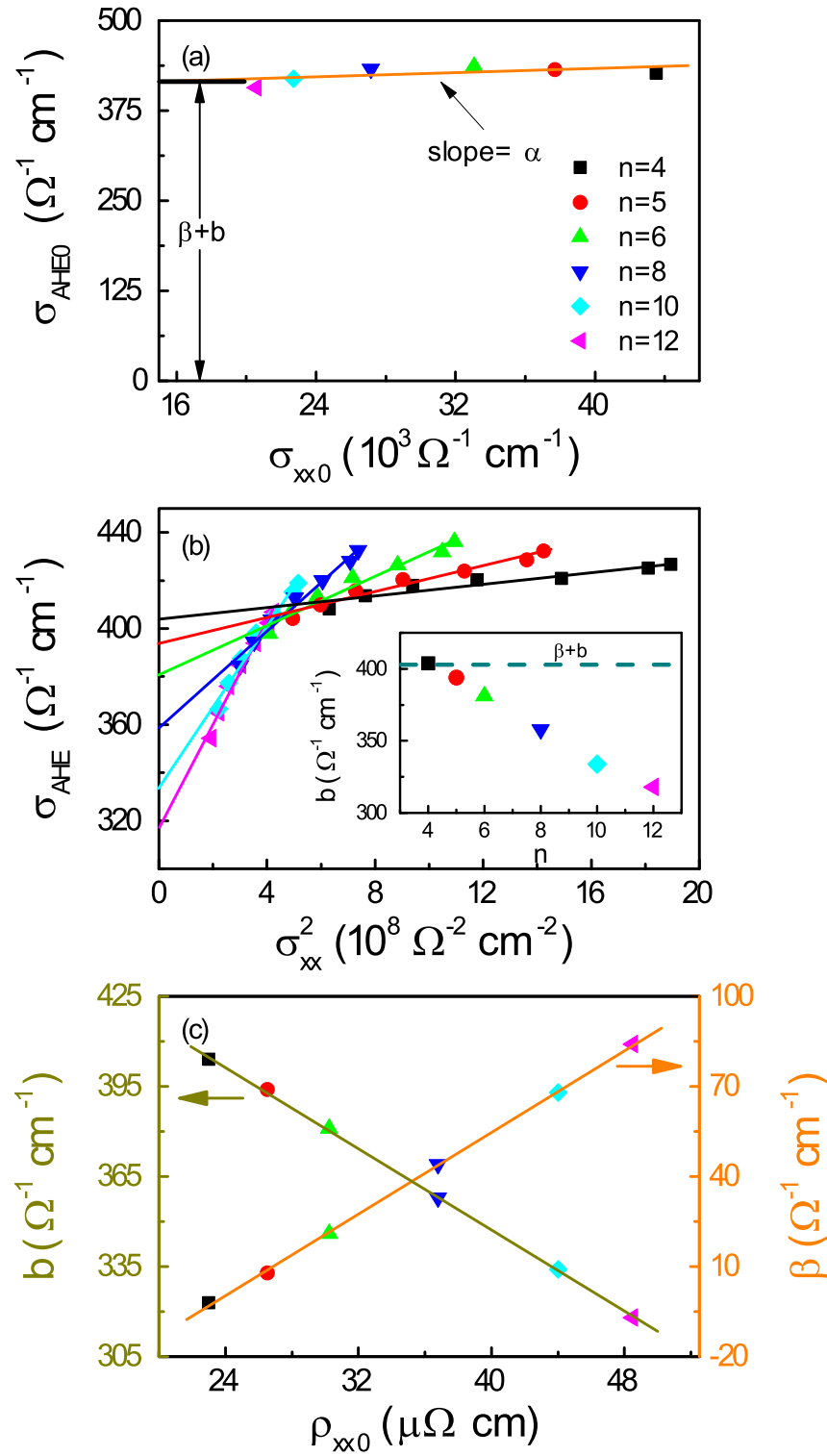

FIG. 8. (a) $\sigma_{\mathrm{AHE} 0} \sim \sigma_{x x 0}$ curve measured at $10 \mathrm{~K}$. The legend is also applied to (b) and (c). (b) $\sigma_{\mathrm{AHE}} \sim \sigma_{x x}^{2}$ curves for $n=4-12$ samples. The inset shows the intercept $(b)$ values for different $n$. (c) $b \sim \rho_{x x 0}$ and $\beta \sim \rho_{x x 0}$ curves. The solid lines indicate the linear fittings to each curve.

found that the relative contributions of the intrinsic mechanism and the side-jump mechanism depend on $\rho_{x x 0}$ which mainly comes from interfacial scattering, suggesting that interfacial scattering suppresses the intrinsic contribution and gives rise to the side-jump contribution. Therefore, interfacial scattering plays a different role than surface scattering. Another interesting feature is that, although the ratio of the intrinsic mechanism to the side-jump mechanism, $b / \beta$, decreases with increasing $\rho_{x x 0}$ (or scattering), the contribution of the intrinsic mechanism dominates the entire longitudinal resistivity range and the full temperature range, which is in agreement with the results reported in Ref. [9]. 


\section{CONCLUSIONS}

In summary, we designed and fabricated a family of multilayered $\left(\mathrm{Fe}_{(36 / n) \mathrm{nm}} / \mathrm{Au}_{(12 / n) \mathrm{nm}}\right)_{n}$ samples to study the AHE. We performed transport property measurements under different magnetic fields in a wide temperature range from 10 to $310 \mathrm{~K}$. We observed different scaling relations in different samples. Our quantitative analysis showed that the interfacial scattering suppresses the contribution of the intrinsic mechanism and gives rise to side-jump contributions. In the search for large spin Hall and anomalous Hall effects, significant attention has been brought to the intrinsic [31] and skew-scattering [32,33] mechanisms. In comparison, the side-jump mechanism has received only little attention lately. Our findings suggest that interfacial scattering can promote the side-jump mechanism which is the source of the AHE and open interesting avenues for the design of this phenomenon in multilayered structures.

\section{ACKNOWLEDGMENT}

The research reported in this publication was supported by funding from King Abdullah University of Science and Technology (KAUST).
[1] E. H. Hall, Philos. Mag. 12, 157 (1881).

[2] R. Karplus and J. M. Luttinger, Phys. Rev. 95, 1154 (1954).

[3] J. Smit, Physica 21, 877 (1955); 24, 39 (1958).

[4] L. Berger, Phys. Rev. B 2, 4559 (1970).

[5] W. J. Xu, B. Zhang, Q. X. Wang, W. B. Mi, Z. Wang, W. Li, R. H. Yu, and X. X. Zhang, Phys. Rev. B 83, 205311 (2011).

[6] H. Liu, F. K. Lee, R. K. Zheng, X. X. Zhang, and O. K. C. Tsui, Phys. Rev. B 70, 224431 (2004).

[7] F. Zhang, F. S. Wen, Y. F. Lü, W. Li, and Y. F. Lu, J. Appl. Phys. 110, 033921 (2011).

[8] Z. B. Guo, W. B. Mi, R. O. Aboljadayel, B. Zhang, Q. Zhang, P. G. Barba, A. Manchon, and X. X. Zhang, Phys. Rev. B 86, 104433 (2012).

[9] Y. Tian, L. Ye, and X. F. Jin, Phys. Rev. Lett. 103, 087206 (2009).

[10] L. Ye, Y. Tian, X. F. Jin, and D. Xiao, Phys. Rev. B 85, 220403(R) (2012).

[11] D. Z. Hou, Y. F. Li, D. H. Wei, D. Tian, L. Wu, and X. F. Jin, J. Phys.: Condens. Matter 24, 482001 (2012).

[12] S. F. Zhang, Phys. Rev. B 51, 3632 (1995).

[13] S. N. Song, C. Sellers, and J. B. Ketterson, Appl. Phys. Lett. 59, 479 (1991).

[14] P. Xiong, G. Xiao, J. Q. Wang, J. Q. Xiao, J. S. Jiang, and C. L. Chien, Phys. Rev. Lett. 69, 3220 (1992).

[15] W. J. Xu, B. Zhang, Z. X. Liu, Z. Wang, W. Li, Z. B. Wu, R. H. Yu, and X. X. Zhang, EPL 90, 27004 (2010).

[16] P. Zhang, W. W. Lin, D. Wu, Z. S. Jiang, and H. Sang, J. Appl. Phys. 115, 063908 (2014).

[17] P. Zhang, K. X. Xie, W. W. Lin, D. Wu, and H. Sang, Appl. Phys. Lett. 104, 082404 (2014).

[18] D. Z. Hou, G. Su, Y. Tian, X. F. Jin, S. Y. A. Yang, and Q. Niu, Phys. Rev. Lett. 114, 217203 (2015).
[19] D. Rosenblatt, M. Karpovski, and A. Gerber, Appl. Phys. Lett 96, 022512 (2010).

[20] C. Borchers, P. Troche, C. Herweg, and J. Hoffmann, J. Mater. Sci. 37, 731 (2002).

[21] Y. S. Yu, H. B. Li, W. L. Li, M. Liu, Y. M. Zhang, and W. D. Fei, J. Magn. Magn. Mater. 322, 1770 (2010).

[22] M. Reghu, C. O. Yoon, D. Moses, and A. J. Heeger, in Handbook of Conducting Polymers, edited by T. A. Skotheim, R. L. Elsenbaumer, and J. R. Reynolds (Marcel Dekker, New York, 1996), p.27.

[23] S. S. Das and M. S. Kumar, J. Phys. D: Appl. Phys. 46, 375003 (2013).

[24] G. Xiao, J. Q. Wang, and P. Xiong, IEEE Trans. Magn. 29, 2694 (1993).

[25] B. A. Aronzon, E. Z. Meilikhov, V. V. Rylkov, A. N. Lagarkov, M. A. Sedova, I. A. E. Evstuhina, D. Y. Kovalev, N. Negre, M. Goiran, and J. Leotin, Physica B 284-288, 1980 (2000).

[26] B. Zhao and X. Yan, J. Appl. Phys. 81, 4290 (1997).

[27] Z. B. Guo, W. B. Mi, A. Manchon, J. Q. Li, B. Zhang, P. G. Barba, and X. X. Zhang, Appl. Phys. Lett. 102, 062413 (2013).

[28] N. F. Mott, Proc. R. Soc. A 153, 699 (1936).

[29] P. M. Levy, S. Zhang, and A. Fert, Phys. Rev. Lett. 65, 1643 (1990).

[30] R. E. Camley and J. Barnas, Phys. Rev. Lett. 63, 664 (1989).

[31] Y. G. Yao, L. Kleinman, A. H. MacDonald, J. Sinova, T. Jungwirth, D. -S. Wang, E. G. Wang, and Q. Niu, Phys. Rev. Lett. 92, 037204 (2004).

[32] A. Fert and P. M. Levy, Phys. Rev. Lett. 106, 157208 (2011)

[33] Y. Niimi, Y. Kawanishi, D. H. Wei, C. Deranlot, H. X. Yang, M. Chshiev, T. Valet, A. Fert, and Y. Otani, Phys. Rev. Lett. 109, 156602 (2012). 